\title{
THE ROLE OF FRESH VERSUS OLD LEAF DAMAGE IN THE ATTRACTION OF PARASITIC WASPS TO HERBIVORE-INDUCED MAIZE VOLATILES
}

\author{
MARIA ELENA HOBALLAH ${ }^{1}$ and TED C. J. TURLINGS* \\ Laboratory of Animal Ecology and Entomology, Institute of Zoology, University of \\ Neuchâtel, Case postale 2, 2007 Neuchâtel, Switzerland
}

(Received January 10, 2005; revised April 8, 2005; accepted May 1, 2005 )

\begin{abstract}
The odor produced by a plant under herbivore attack is often used by parasitic wasps to locate hosts. Any type of surface damage commonly causes plant leaves to release so-called green leaf volatiles, whereas blends of inducible compounds are more specific for herbivore attack and can vary considerably among plant genotypes. We compared the responses of naïve and experienced parasitoids of the species Cotesia marginiventris and Microplitis rufiventris to volatiles from maize leaves with fresh damage (mainly green leaf volatiles) vs. old damage (mainly terpenoids) in a six-arm olfactometer. These braconid wasps are both solitary endoparasitoids of lepidopteran larvae, but differ in geographical origin and host range. In choice experiments with odor blends from maize plants with fresh damage $v s$. blends from plants with old damage, inexperienced $C$. marginiventris showed a preference for the volatiles from freshly damaged leaves. No such preference was observed for inexperienced M. rufiventris. After an oviposition experience in hosts feeding on maize plants, C. marginiventris females were more attracted by a mixture of volatiles from fresh and old damage. Apparently, C. marginiventris has an innate preference for the odor of freshly damaged leaves, and this preference shifts in favor of a blend containing a mixture of green leaf volatiles plus terpenoids, after experiencing the latter blend in association with hosts. M. rufiventris responded poorly after experience and preferred fresh damage odors. Possibly, after associative learning, this species uses cues that are more directly related with the host presence, such as volatiles from host feces, which were not present in the odor sources offered in the olfactometer. The results demonstrate the complexity of the use of plant
\end{abstract}

* To whom correspondence should be addressed. E-mail: ted.turlings@unine.ch

${ }^{1}$ Current address: Institute of Plant Sciences, University of Bern, Altenbergrain 21, 3013 Bern, Switzerland. 
volatiles by parasitoids and show that different parasitoid species have evolved different strategies to exploit these signals.

Key Words-Cotesia marginiventris, Microplitis rufiventris, Spodoptera littoralis, Zea mays, parasitoid, innate preference, associative learning, induced volatiles, green leaf volatiles, terpenoids, host location.

\section{INTRODUCTION}

Many parasitoid species make use of herbivore-induced volatiles to locate their herbivorous hosts. Various studies on host searching by parasitoids show that plant-provided cues are more important for the location of host habitats than odor cues from the host or host by-products (Turlings et al., 1990; Steinberg et al., 1993; McCall et al., 1993; Agelopoulos and Keller, 1994; Geervliet et al., 1994; Mattiacci et al., 1994; Takabayashi et al., 1995; Fukushima et al., 2002), but it remains largely unknown which compounds are the most crucial for the attraction.

That volatile blends can differ in their attractiveness is evident from various studies that show differential attraction mediated by odors of different plant species attacked by the same herbivore (Drost et al., 1988; McAuslane et al., 1991; Geervliet et al., 1996; De Moraes and Lewis, 1999). Also within one plant species, however, there can be considerable variation in attractiveness among genotypes. For instance, Cotesia marginiventris, a generalist parasitoid that attacks early instars of many Lepidoptera, prefers the caterpillar-induced odor of some specific maize varieties over others, and this preference is not simply a matter of differences in quantity of volatiles emitted (Hoballah Fritzsche et al., 2002). Moreover, several parasitoids show variable responses to odors emitted by the same plant species attacked by different herbivores, such as Microplitis croceipes (Zanen and Cardé, 1991), Cotesia sesamiae and Cotesia flavipes, (Ngi-Song et al., 1996), and Cotesia glomerata (Geervliet et al., 1997). Specialist parasitoids may use their ability to make such distinctions to focus only on plants that carry their specific hosts, as was found for Aphidius ervi, an aphid parasitoid with a limited host range (Du et al., 1998; Powell et al., 1998), and Cardiochiles nigriceps, which can only successfully develop in larvae of Heliothis virescens (De Moraes et al., 1998; De Moraes and Lewis, 1999). Equally intriguing is the observation that Cotesia kariyai can distinguish among odors produced by the same plant species fed upon by different larval stages of their respective host, Pseudaletia separata (Takabayashi et al., 1995). However, Microplitis rufiventris, which can also only develop in early instars of some Lepidoptera, does not show a preference for maize damaged by a particular larval stage of Spodoptera littoralis (Gouinguené and Turlings, 2003). 
A review by Dicke (1999) shows that evidence for specificity in signals is highly variable, suggesting that different parasitoid species employ different strategies to exploit plant-provided signals to find hosts. As proposed by Vet and Dicke (1992), parasitoids use volatiles emitted by host-damaged plants rather than volatiles from the hosts themselves because plant volatiles are released in much larger quantities and are easier to detect. However, damaged plants are expected to provide limited information on the suitability as host of an herbivore that has been damaging it. This problem is partially solved by the ability of parasitoids to learn by association (Vet and Dicke, 1992). For various parasitoids, it has been shown that they learn to respond to a specific odor when they perceive it during contact with the host or host feces (Lewis and Tumlinson, 1988; Vet and Groenewold, 1990; Turlings et al., 1993; Vet et al., 1995). This ability may allow the wasps to learn subtle differences between odor blends and thus focus on odors that are more reliably associated with the presence of suitable hosts. C. marginiventris, for example, shifts its preference in favor of plant odors that it has experienced during an encounter with a host (Turlings et al., 1989, 1993). This wasp can even learn to distinguish between the odors released by maize plants fed on by two closely related Spodoptera species (Turlings et al., 1993).

What do generalist parasitoids such as $C$. marginiventris respond to before they have found their first host? One possibility is that they learn during emergence from the cocoon and initially focus on the same cues that guided their mother to hosts. Such early adult learning was found, for example, in Microplitis demolitor (Hérard et al., 1988), Cotesia plutella (Bogahawatte and Van Emden, 1996), and Aphidius colemani (Douloumpaka and Van Emden, 2003). A second possibility is that, as they emerge from the cocoon, the wasps will first cue on general plant odors, and that they will learn plant odors that are associated with the specific host only as soon as the first host is encountered and parasitized. Independent of plant genotype, the so-called green leafy volatiles (6-carbon aldehydes, alcohols, and acetates) are common and typically released from freshly damaged leaves, whereas herbivore-induced volatiles, such as blends of terpenoids, are more indicative of actual herbivore damage, and their composition varies among plant genotypes.

The aim of the current study was to test the hypothesis that inexperienced C. marginiventris females initially prefer blends with common fresh damage volatiles, and experienced females shift their preference toward a more specific blend. A second parasitoid, Microplitis rufiventris (Hymenoptera: Braconidae) with a more restricted host range, was included to determine a possible difference in exploitation of plant volatiles by generalists and specialists. These two parasitoids originate from different continents (near-arctic region and Northeast Africa, respectively). C. marginiventris parasitizes at least 25 Lepidoptera (including Spodoptera spp., Helicoverpa spp.) (Maes, 1989), 
whereas M. rufiventris has been found only on Spodoptera littoralis, Spodoptera exigua, and Heliothis armigera (Hegazi and El-Minshawy, 1979). The hosts for both parasitoids can be found on many of the same crop plants (cotton, maize, cabbage, sweet potato, tomato, and other crops), implying that there is substantial overlap in potential plant cues that they can use.

Here, we used damaged maize plants as the odor sources. In all cases, plants were mechanically damaged and treated with caterpillar regurgitant. Such treatment results in the immediate release of several compounds, such as the green leafy volatiles, whereas the herbivore-inducible compounds, comprising mainly terpenoids and some aromatics, predominantly indole, appear at the earliest several hours after initial damage (Turlings et al., 1998). By offering the choice between odor of freshly damaged plants and the odor of plants with old damage to the wasps, the relative importance of the two volatile groups as attractants could be tested.

\section{METHODS AND MATERIALS}

Plants and Insects. Two-wk-old maize plants (var. Delprim) were used for all experiments. Two seeds per pot $(200 \mathrm{ml}, 6 \mathrm{~cm}$ high $)$ were planted in commercial soil (COOP, Switzerland) and grown in a climate chamber $\left(23^{\circ} \mathrm{C}\right.$, $60 \%$ r.h., 16D:8L, 50,000 $1 \mathrm{~m} \mathrm{~m}^{-2}$ ). The day before an experiment was started, plants were transferred in glass pots that fit the olfactometer $(250 \mathrm{ml}, 4.5 \mathrm{~cm}$ diam, $11 \mathrm{~cm}$ high).

Spodoptera littoralis eggs were obtained from Syngenta (Stein, Switzerland). Rearing methods for hosts and parasitoids are described by Fritzsche Hoballah and Turlings (2001). Two parasitoids were used for the study, $C$. marginiventris and M. rufiventris. C. marginiventris was obtained from the USDA-ARS, Biological Control and Mass Rearing Research Unit (MS, USA), and $M$. rufiventris from the Faculty of Agriculture, Alexandria University (Egypt). Parasitized larvae and adult $C$. marginiventris were kept in an incubator $\left(25^{\circ} \mathrm{C}\right.$ and $\left.16 \mathrm{~L}: 8 \mathrm{D}\right)$ until the day of the experiment, whereas parasitized larvae and adults of $M$. rufiventris were kept in the laboratory under ambient light and temperature conditions. Female parasitoids were used for the assays when they were $2-5 \mathrm{~d}$ old.

Six-arm Olfactometer Bioassay (Inexperienced Wasps). The six-arm olfactometer is described by Turlings et al. (2004). This system exposes wasps to six air streams entering a central choice chamber. Each stream, entering the chamber via a glass tube, can carry a different odor. In our bioassays, only three odor sources were offered to the parasitoids and each arm with odorous air stream was alternated with a neighboring arm carrying a stream of clean air. Charcoal filters purified and a bubbler humidified the air that was then pushed 
into each odor source vessel at a rate of $1.21 \mathrm{~min}^{-1}$. During each bioassay, a portion of each odor was collected by pulling air from each vessel at a rate of $0.6 \mathrm{ml} \mathrm{min}{ }^{-1}$ through Super-Q traps (see Heath and Manukian, 1992) that were attached to the vessels just above the treated pants (for details, see Turlings et al., 2004).

To induce volatile emissions in the plants that served as odor sources, we scratched the underside of two leaves over an area of $2-4 \mathrm{~cm}^{2}$ on both sides of the central vein of the leaf with a razor blade, after which $10 \mu \mathrm{l}$ of S. littoralis regurgitant were applied on each damaged site. The regurgitant had been collected from fourth and fifth instar $S$. littoralis caterpillars that had fed on maize leaves (var. Delprim) and had been stored at $-70^{\circ} \mathrm{C}$ until just before use (for details, see Turlings et al., 1998). After treatment, the plants were placed under three fluorescent lamps (Sylvania standard F36W 133-T8 cool white, $5,000 \mathrm{~lm} \mathrm{~m} \mathrm{~m}^{-2}$ at pot height) until used in bioassays.

Two combinations of three odor sources were offered as choice to inexperienced wasps. The combination of odors consisted of: (1) two maize plants treated $30 \mathrm{~min}$ before (fresh-fresh), two maize plants treated $6 \mathrm{hr}$ before (old-old), and one plant treated $30 \mathrm{~min}$ before plus one $6 \mathrm{hr}$ before (fresh-old) assaying; (2) two maize plants treated $30 \mathrm{~min}$ before (fresh-fresh), two maize plants treated $6 \mathrm{hr}$ before (old-old), and two maize plants left unharmed, but treated with $20 \mu \mathrm{l}$ of $S$. littoralis regurgitant on the leaf surface (spit) before assaying.

Wasps were released in groups of six into the choice chamber, and after $30 \mathrm{~min}$, the wasps' choices for a particular arm were noted. Wasps that did not enter an arm after 30 min were considered a "no choice." For each replication day, the position of the odor sources was shifted. Between two and three replications per wasp species were carried out per day. On almost all replication days, both groups were tested using same odor sources (statistical tests confirmed that there was no influence of time of release even if some test days lasted $3 \mathrm{hr}$ with three replications for each wasp species). After each experimental day, the olfactometer was washed with running tap water and soap, subsequently rinsed with acetone and pentane, and air-dried before being placed in the oven at $250^{\circ} \mathrm{C}$ for $3 \mathrm{hr}$. The Teflon tubes connecting the glass parts of the olfactometer were also rinsed with acetone and pentane.

A log-linear model fitted for the expected distribution of the wasps within the olfactometer (quasi-Poisson model) that do not conform to simple variance assumptions was used in statistical tests for odor preferences (Turlings et al., 2004). The numbers of wasps that chose one of the arms containing an odor source (three odor treatments plus three arms containing pure air as fourth treatment) for each released group (six wasps) were used as a replication. The numbers of replications for each test are given in the figure legends. We used the general linear model coefficients to determine preferences for one of the 
treatments. $P$ and $F$ values are given only for the analysis of preference among the three odor cue treatments (not including the number of wasps that entered in the arm offering only clean air as odor cue). The software package $\mathrm{R}$ http:// www.imsv.unibe.ch/cran) was used to test the model fitted by quasi-likelihood estimation, and its relative adequacy was assessed through likelihood ratio statistics and examination of residuals.

Six-arm Olfactometer Bioassay (Experienced Wasps). To provide them experience, the wasps were placed in a plastic box $(9 \mathrm{~cm}$ top diam, $8.5 \mathrm{~cm}$ bottom diam, $3.5 \mathrm{~cm}$ high) containing maize leaves (var. Delprim) on which larvae of $S$. littoralis had been feeding for $24 \mathrm{hr}$. After females parasitized one or two larvae, they were considered experienced. This treatment is known to increase the responsiveness of parasitoids to the odors they perceive during the experience (Turlings et al., 1993). We gave only one oviposition experience to $M$. rufiventris to obtain approximately the same period of contact with the leaf-host complex for both parasitoid species, because $M$. rufiventris needs more time than $C$. marginiventris to parasitize the same number of hosts (Hoballah, personal observation).

Only one odor source combination was tested with experienced wasps: two maize plants treated $30 \mathrm{~min}$ before (fresh-fresh), two maize plants treated $6 \mathrm{hr}$ before (old-old), and one plant treated $30 \mathrm{~min}$ before and one $6 \mathrm{hr}$ before (fresh-old) assaying. Treatment of plants, odor collections, and statistical analyses were the same as for the bioassays with inexperienced wasps.

Volatile Collections and Analyses. Traps containing Super-Q (25 mg, 80/ 100 mesh; Alltech Associates, Inc., Deerfield State, USA) were used to collect odors from various treatments during the olfactometer experiments. Collections started $20 \mathrm{~min}$ before an olfactometer experiment and ended after a period of $3 \mathrm{hr}$ had elapsed. After each collection, traps were rinsed with $150 \mu$ l methylene chloride. Two hundred ng of $n$-octane and nonyl acetate were added as internal standards. Three- $\mu$ l aliquots of the samples were injected on column, with an automated injection system, into a Hewlett Packard model HP 6890 gas chromatograph equipped with a flame ionization detector. The HP-1 capillary column (30 m, $0.25 \mathrm{~mm}$ internal diam, $0.25 \mu \mathrm{m}$ film thickness; Hewlett-Packard Company, USA) was kept at $50^{\circ} \mathrm{C}$ for $3 \mathrm{~min}$ and then programmed at $8^{\circ} \mathrm{C}$ $\min ^{-1}$ to $230^{\circ} \mathrm{C}$, where it was maintained for $9.5 \mathrm{~min}$. The column was preceded by a deactivated retention gap $(10 \mathrm{~m}, 0.25 \mathrm{~mm}$ internal diam; Alltech Associates) and a deactivated precolumn $(30 \mathrm{~cm}, 0.53 \mathrm{~mm}$ internal diam; Alltech Associates). Helium (24 $\mathrm{cm} \mathrm{sec}^{-1}$ ) was used as carrier gas. HP GC Chemstation software was used to quantify all major components by comparison to the known quantity of internal standards. ANOVA and Student-Newman-Keuls post-hoc test were used to compare the quantities of collected volatiles (total of all compounds combined as well as of single compounds). 

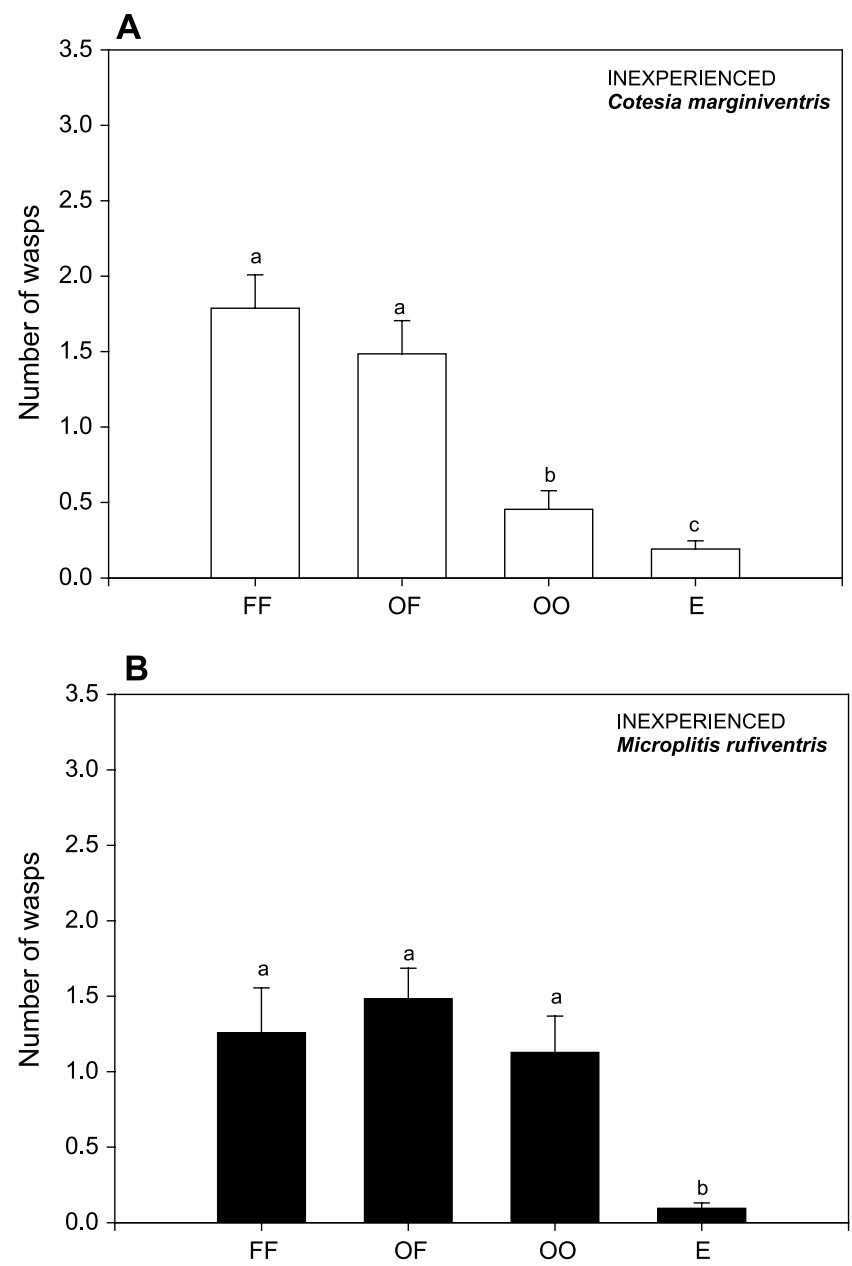

FIG. 1. Mean $( \pm \mathrm{SE})$ of inexperienced Cotesia marginiventris (A, 33 replications, $26.8 \%$ "no choice") and Microplitis rufiventris (B, 31 replications, 30.1\% "no choice") females that were attracted to a specific source in a six-arm olfactometer. FF: Two maize plants treated $30 \mathrm{~min}$; OF: one plant treated $30 \mathrm{~min}$ and one plant treated $6 \mathrm{hr}$; OO: two plants treated $6 \mathrm{hr}$, respectively, before experiment started; E: clean air, mean $( \pm \mathrm{SE})$ for the three empty olfactometer arms. Damage treatments consisted of scratching two leaves of a plant and adding Spodoptera littoralis regurgitant on the damaged sites. Different letters above bars indicate significant differences among total numbers of wasp that chose a particular odor source including clean air (preference for one of the three odor treatments: $\mathbf{A}, P<0.001, F=15.87, d f=2 ; \mathbf{B}, P=0.608, F=0.50, d f=2)$. 


\section{RESULTS}

Six-arm Olfactometer Bioassays (Inexperienced Wasps). C. marginiventris preferred the "fresh-fresh" and "fresh-old" odor to the odor of the "old-old" treatment, meaning that volatiles released after fresh damage are important for initial attraction (Figure 1A). Still, the "old-old" odor was more attractive than clean air (Figure 1A). In contrast, inexperienced $M$. rufiventris was equally attracted to all three treatments (Figure 1B). Both wasps rarely entered the three arms of the olfactometer carrying pure air, but almost one third of the wasps did not choose any arm (Figure 1).

The total amount of volatiles collected from the plants treated in different ways was not significantly different (mean \pm SE for fresh-fresh: $613.6 \pm 57 \mathrm{ng}$, fresh-old: $584.5 \pm 72 \mathrm{ng}$, old-old: $610.6 \pm 97 \mathrm{ng}$, ANOVA $P=0.958, F=$ 0.043). The odors collected during the olfactometer experiences show a

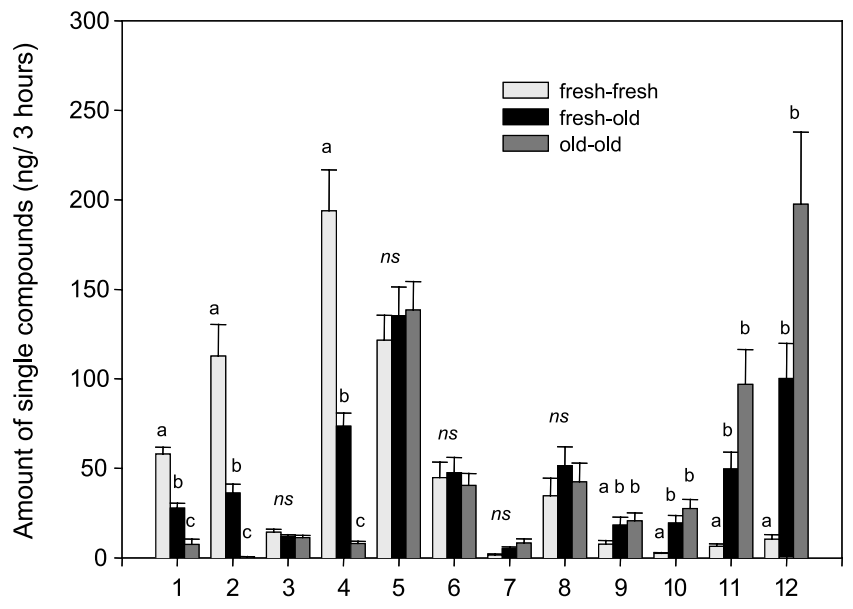

FIG. 2. Mean $( \pm \mathrm{SE})$ amounts of major single volatile compounds of a blend collected for $3 \mathrm{hr}$ from: two maize plants treated $30 \mathrm{~min}$ (fresh-fresh), one plant treated $30 \mathrm{~min}$ and one plant treated $6 \mathrm{hr}$ (fresh-old), and two plants treated $6 \mathrm{hr}$ (old-old) before experiments in a six-arm olfactometer started. Damage treatments consisted of scratching two leaves of a plant and adding Spodoptera littoralis regurgitant on the damaged sites. Different letters above bars indicate significant differences in amount of single compounds collected among treatments and $n s$ indicate no significant difference among treatments. This graph includes the volatile collections of all experiments carried out with those treatments (experiments with inexperienced and experienced wasps, $N=12$ ). 1, (E)-2-Hexenal; 2, ( $Z$ )-3-hexenol; 3, $\beta$-myrcene; 4, $(Z)$-3-hexenyl acetate; 5, linalool; 6, (E)-4,8-dimethyl-1,3,7-nonatriene; 7, phenethyl acetate; 8, 1-H-indole; 9, geranyl acetate; 10, $(E)$ - $\beta$-caryophyllene; 11, $(E)$ - $\alpha$-bergamotene; 12, $(E)$ - $\beta$-farnesene. 

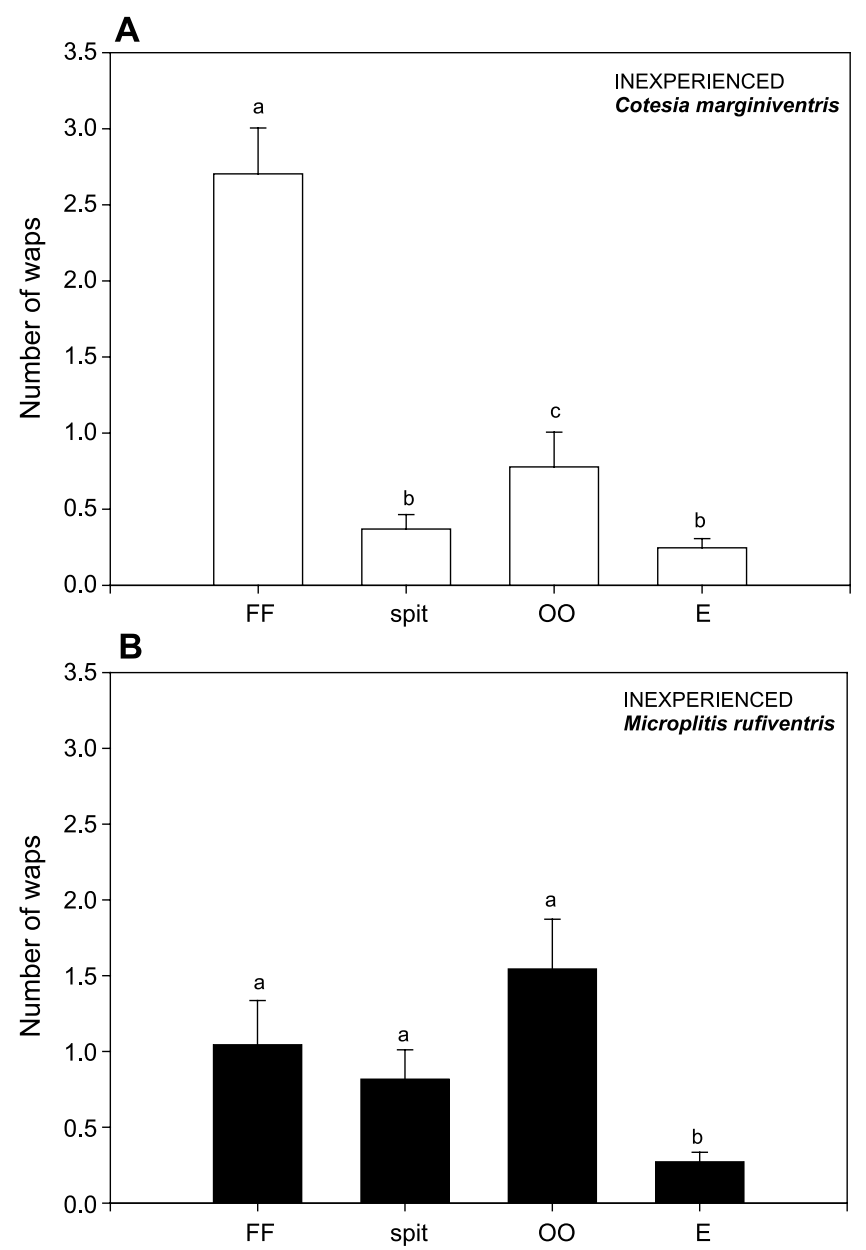

FIG. 3. Mean $( \pm \mathrm{SE})$ of inexperienced Cotesia marginiventris (A, 27 replicates, $22.8 \%$ "no choice") and Microplitis rufiventris ( B, 22 replicates, $27.3 \%$ "no choice") females that were attracted to a specific source in a six-arm olfactometer. FF: Two maize plants treated 30 min; spit: two undamaged plants on which Spodoptera littoralis regurgitant was smeared; OO: two plants treated $6 \mathrm{hr}$ before experiment started; E: clean air, mean $( \pm \mathrm{SE}$ ) for the three empty olfactometer arms. Damage treatments consisted of scratching two leaves of a plant and adding Spodoptera littoralis regurgitant on the damaged sites. Different letters above bars indicate significant differences among the numbers of wasp that chose a particular odor source including clean air (preference for one of the three odor treatments: $\mathbf{A}, P<0.001, F=27.94, d f=2 ; \mathbf{B}, P=0.169, F=1.83, d f=2$ ). 
difference in the amounts of green leaf volatiles and terpenoids emitted by the treatments, with the "fresh-old" treatments showing intermediate release of both groups (Figure 2).

In the experiment where the wasps had a choice between (a) the odor of two freshly damaged plants, (b) the odor of two plants with 6-hr-old damage, and (c) the odor of two undamaged plants with regurgitant smeared on the leaves, C. marginiventris preferred the fresh damage (Figure 3A), whereas inexperienced M. rufiventris again showed no significant preference (Figure 3B). The relatively high number of $M$. rufiventris choosing the odor of undamaged plants treated with regurgitant was unexpected, because the amount of all major compounds released from this treatment was significantly lower than for the other two treatments (Figure 4).

Six-arm Olfactometer Bioassays (Experienced Wasps). When C. marginiventris wasps were given an oviposition experience on $S$. littoralis caterpillars feeding on maize (var. Delprim) leaves, their preference changed. Experienced

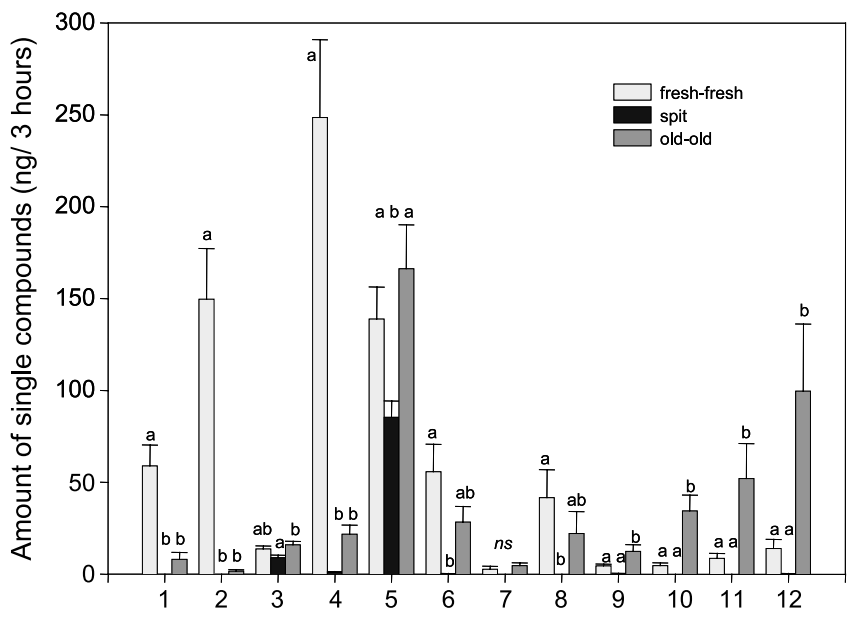

FIG. 4. Mean ( \pm SE $)$ amounts of major single volatile compounds of a blend collected for $3 \mathrm{hr}$ from two maize plants treated $30 \mathrm{~min}$ (fresh-fresh), two plants smeared with Spodoptera littoralis regurgitant (spit), and two plants treated $6 \mathrm{hr}$ (old-old), before experiments in a six-arm olfactometer started. Damage treatments consisted of scratching two leaves of a plant and adding $S$. littoralis regurgitant on the damaged sites. Different letters above bars indicate significant differences in amount of single compounds collected among treatments and $n s$ indicate no significant difference among treatments. 1, (E)-2-Hexenal; 2, ( $Z$ )-3-hexenol; 3, $\beta$-myrcene; 4, $(Z)$-3-hexenyl acetate; 5, linalool; 6, (E)-4,8-dimethyl-1,3,7-nonatriene; 7, phenethyl acetate; 8, 1-H-indole; 9, geranyl acetate; 10, $(E)$ - $\beta$-caryophyllene; 11, $(E)$ - $\alpha$-bergamotene; 12, $(E)$ - $\beta$-farnesene. 

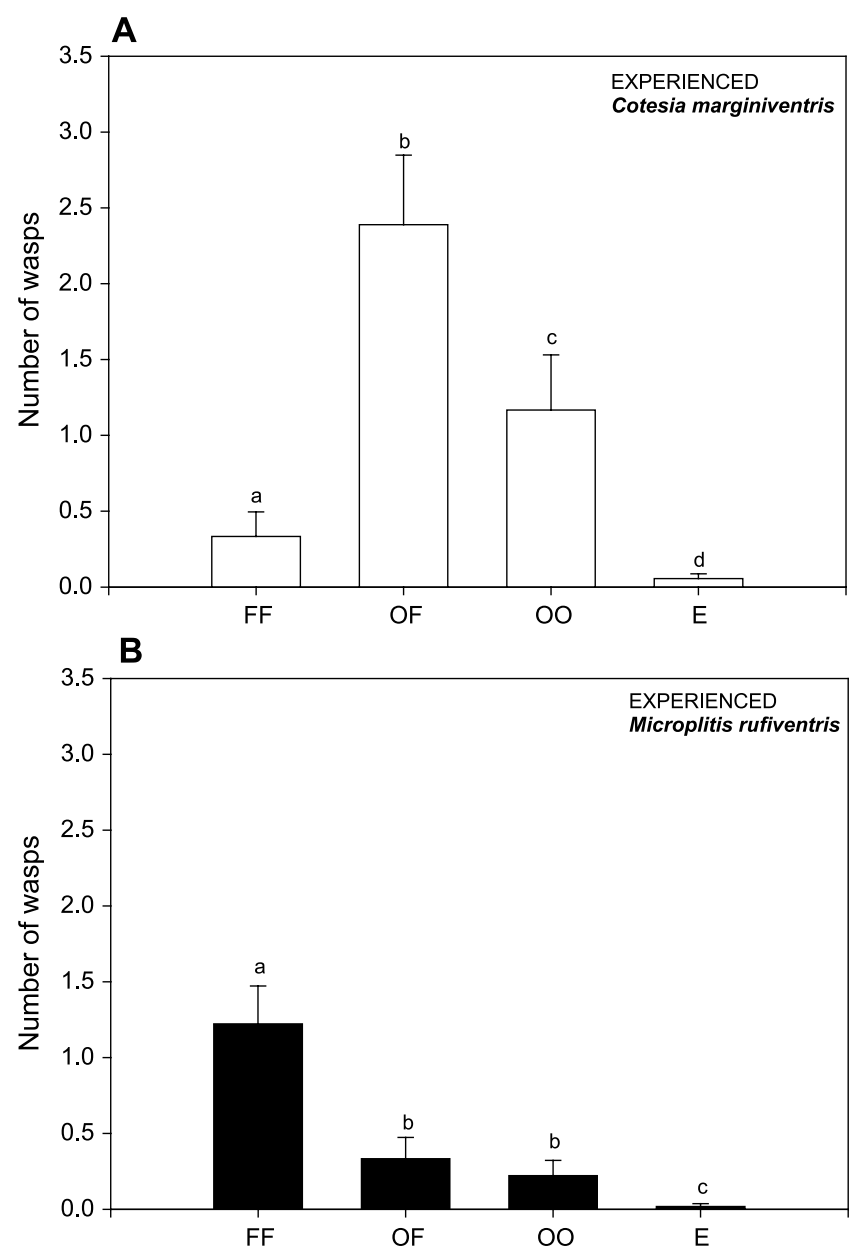

FIG. 5. Mean $( \pm \mathrm{SE})$ of experienced Cotesia marginiventris (A, 18 replicates, $32.4 \%$ "no choice") and Microplitis rufiventris females (B, 18 replicates, $69.4 \%$ "no choice") that were attracted to a specific source in a six-arm olfactometer. Experience consisted of an oviposition in a host on maize leaves that had been damaged by hosts for $24 \mathrm{hr}$. FF: Two maize plants treated $30 \mathrm{~min}$; OF: one plant treated $30 \mathrm{~min}$ and one plant treated 6 hr; OO: two plants treated $6 \mathrm{hr}$, respectively, before an experiment started; E: clean air, mean $( \pm \mathrm{SE})$ for the three empty olfactometer arms. Damage treatment consisted of scratching two leaves of a plant and adding Spodoptera littoralis regurgitant on the damaged sites. Different letters above bars indicate significant differences among total numbers of wasp that chose a particular odor source including clean air (preference for one of the three odor treatments: $\mathbf{A}, P<0.001, F=9.49, d f=2 ; \mathbf{B}, P<0.001, F=9.16$, $d f=2)$. 
C. marginiventris females significantly preferred the complete blend (fresh-old) to blends containing larger amounts of green leaf volatiles (fresh-fresh), or terpenoids (old-old) (Figure 5A). Surprisingly, the majority of experienced $M$. rufiventris did not make any choice, and among those that did, the majority preferred the odor source containing more green leaf volatiles (Figure 5B). As was the case for inexperienced wasps, experienced wasps of both species rarely entered one of the three arms of the olfactometer that carried clean air (Figure 5).

\section{DISCUSSION}

Confirming our hypothesis, inexperienced C. marginiventris preferred blends containing a higher proportion of green leaf volatiles, indicating that, as expected, green leaf volatiles or compounds that are simultaneously released are important for initial attraction of inexperienced C. marginiventris. Odor released after recent damage is important also in attracting naïve C. marginiventris to cotton plants (Cortesero et al., 1997). Several parasitoids orient to individual green leaf volatile compounds in an olfactometer or flight tunnel tests (Whitman and Eller, 1990; Wickremasinghe and Van Emden, 1992; Reddy et al., 2002) or show strong electroantennogram responses to these compounds (Baehrecke et al., 1989; Li et al., 1992). However, there are exceptions. For instance, artificially damaged plants (an unspecific blend containing some green leaf volatiles) elicited little response in naïve Cotesia kariyai (Fukushima et al., 2002). The responses of $M$. rufiventris observed here show that for this species, green leaf volatiles are not crucial for initial attraction. In fact, inexperienced M. rufiventris responded well, but did not distinguish among the different odor blends offered. Surprisingly, even the undamaged plants that were treated with regurgitant were attractive to this wasp. It should be noted that several maize varieties, including Delprim, release some volatiles when they are not damaged (Turlings et al., 1998). It is possible that these compounds, which include linalool, are important for the attraction of M. rufiventris, and that some compounds in the other blends repel this wasp. A closely related species, Microplitis croceipes, is also equally attracted to the odors of artificially damaged plants and undamaged cotton plants (Röse et al., 1998). Apparently, these specialized wasps use cues other than the general volatiles emitted by the damaged plant, cues that are more specifically linked with their hosts. This also seems to be the case for the specialist Cotesia rubecula, which is more attracted by the odors of Arabidopsis thaliana damaged by its host (Pieris rapae) than by artificially damaged $A$. thaliana (van Poecke et al., 2001), and more by host-damaged plants compared to jasmonic acid treated plants (van Poecke and Dicke, 2002). 
That inexperienced C. marginiventris and M. rufiventris, both solitary endoparasitic braconids, have different odor preferences may reflect differences in host location strategies between generalists and specialists. The generalist C. marginiventris oriented primarily toward odors released by freshly damaged plants, whereas the specialist $M$. rufiventris oriented to all treatment plants equally. A difference between the two species was also apparent after experience. C. marginiventris that experienced an oviposition on plants that had been damaged by host larvae overnight preferred the complete blend (fresh-old). Because the experience of oviposition was associated with the entire complex, host-damaged leaves, host larvae, and host by-products, it was to be expected that the wasps would be more attracted to the complete blend. Surprising was the preference of experienced $M$. rufiventris females for the blends containing a higher proportion of green leaf volatiles, as was the high proportion of experienced wasps that did not make a choice. It is possible that the experienced M. rufiventris searched for odors that are more specifically associated with the presence of hosts and that were not present among the cues offered.

It should be pointed out that we have emphasized green leaf volatiles and terpenoids as the groups of compounds associated with fresh and old damage; however, many other compounds are released in minor quantities, and these compounds may play a role in the attraction of the wasps as well. Electroantennogram studies have shown that some minor compounds elicit strong antennal responses in these parasitoids (Gouinguené et al., 2005), and current attempts to isolate and identify key attractants for C. marginiventris indicate an important role for minor compounds (M. D'Alessandro and T.C.J. Turlings, unpublished data). This does not invalidate the main conclusion from this study - that some generalist parasitoids use volatiles from fresh damage as general cues, and that truly herbivore-induced compounds, or other compounds directly associated with host presence, become more important after the wasps have experienced them while encountering hosts. Moreover, it can be concluded that parasitoids with comparable biologies, but different host ranges, employ different strategies in their use of plant-provided cues to locate hosts. Further insight into the host specificity and the circumstances under which wasps have to forage may provide explanations for these differences.

Acknowledgments - We thank Liselore Roelfstra and Yves Borcard for parasitoid rearing. Parasitoids with which we started our lab colonies were supplied by Donald Nordlund $(C$. marginiventris) and Esmat Hegazi (M. rufiventris). We also thank Syngenta (Stein, Switzerland) for the weekly shipment of Spodoptera littoralis eggs and caterpillars. Alex Aebi, Louis Felix Bersier, Marco D’Alessandro, Thomas Degen, Martine Rahier, and Cristina Tamò contributed with stimulating discussions and/or comments on the initial manuscript. We are grateful to Matthias Held, Ingrid Ricard, and Anthony Davison for the log-linear model construction and help with statistical analyses. This study was supported by grants from the Swiss Centre of International Agriculture (ZIL, ETH Zurich) and the Swiss National Science Foundation (grants 31-46237-95 and 31-44459-95). 


\section{REFERENCES}

Agelopoulos, N. G. and Keller, M. A. 1994. Plant-natural enemy association in the tritrophic system, Cotesia rubecula-Pieris rapae-Brassicaceae (Cruciferae): I. Sources of infochemicals. J. Chem. Ecol. 20:1725-1734.

BAeHRECKe, E. H., Williams, H. J., and Vinson, S. B. 1989. Electroantennogram responses of Campoletis sonorensis (Hymenoptera: Ichneumonidae) to chemicals in cotton (Gossypium hirsutum L.). J. Chem. Ecol. 15:37-45.

Bogahawatte, C. N. L. and Van EmDen, H. F. 1996. The influence of the host plant of diamondback moth (Plutella xylostella) on the plant preferences of its parasitoid Cotesia plutellae in Sri Lanka. Physiol. Entomol. 21:93-96.

Cortesero, A. M., De Moraes, C. M., Stapel, J. O., Tumlinson, J. H., and Lewis, W. J. 1997. Comparisons and contrasts in host-foraging strategies of two larval parasitoids with different degrees of specificity. J. Chem. Ecol. 23:1589-1606.

DE Moraes, C. and LeWIS, W. J. 1999. Analyses of two parasitoids with convergent foraging strategies. J. Insect. Behav. 12:571-583.

de Moraes, C. M., Lewis, W. J., Paré, P. W., Alborn, H. T., and Tumlinson, J. H. 1998. Herbivore-infested plants selectively attract parasitoids. Nature 393:570-573.

DICKE, M. 1999. Are herbivore-induced plant volatiles reliable indicators of herbivore identity to foraging carnivorous arthropods? Entomol. Exp. Appl. 91:131-142.

DOUlOUMPaKa, S. and VAN EMDEN, H. F. 2003. A maternal influence on the conditioning to plant cues of Aphidius colemani Viereck, parasitizing the aphid Myzus persicae Sulzer. Physiol. Entomol. 28:108-113.

Drost, Y. C., Lewis, W. J., and TumLINSON, J. H. 1988. Beneficial arthropod behavior mediated by airborne semiochemicals: V. Influence of rearing method, host plant, and adult experience on host-searching behavior of Microplitis croceipes (Cresson), a larval parasitoid of Heliothis. J. Chem. Ecol. 14:1607-1616.

Du, Y., Poppy, G. M., Powell, W., Pickett, J. A., Wadhams, L. J., and Woodcock, C. M. 1998. Identification of semiochemicals released during aphid feeding that attract parasitoid Aphidius ervi. J. Chem. Ecol. 24:1355-1368.

Fritzsche Hoballah, M. E. and Turlings, T. C. J. 2001. Experimental evidence that plants under caterpillar attack may benefit from attracting parasitoids. Evol. Ecol. Res. 3:1-13.

FUKUSHIMA, J., KAINOH, Y., HONDA, H., and TAKABAYASHI, J. 2002. Learning of herbivore-induced and nonspecific plant volatiles by a parasitoid, Cotesia kariyai. J. Chem. Ecol. 28:579-586.

GeERVLIET, B. F., VET, L. E. M., and DicKE, M. 1994. Volatiles from damaged plants as major cues in long-range host-searching by the specialist parasitoid Cotesia rubecula. Entomol. Exp. Appl. 73:289-297.

GeERVliet, J. B. F., Vet, L. E. M., and Dicke, M. 1996. Innate response of the parasitoids Cotesia glomerata and C. rubecola (Hymenoptera: Braconidae) to volatiles from different plantherbivore complexes. J. Insect Behav. 9:525-538.

Geervliet, J. B. F., Posthumus, M. A., Vet, L. E. M., and Dicke, M. 1997. Comparative analysis of headspace volatiles from different caterpillar-infested or uninfested food plants of Pieris species. J. Chem. Ecol. 23:2935-2954.

Gouinguené, S. and TURLINGS, T. C. G. 2003. Induction of volatile emissions in maize by different larval instars of Spodoptera littoralis. J. Chem. Ecol. 29:145-162.

Gouinguené, S., Pickett, J., Wadhams, L. G., Birkett, M. A., and Turlings, T. C. G. 2005. Antennal electrophysiological responses of three parasitic wasps to the caterpillar induced volatiles from maize (Zea mays mays), cowpea (Gossypium herbaceum) and cotton (Vigna unguiculata). J. Chem. Ecol. 31:1023-1038. 
Heath, R. R. and ManuKian, A. 1992. Development and evaluation of systems to collect volatile semiochemicals from insects and plants using a charcoal-infused medium for air purification. J. Chem. Ecol. 18:1209-1226.

HegaZI, E. M. and El-MinSHAwy, A. M. 1979. Laboratory technique for mass-rearing Microplitis rufiventris Kok. (Braconidae; Hymenoptera), an internal parasite of the cotton leaf-worm Spodoptera littoralis (Boisd.) (Noctuidae; Lepidoptera). Boll. Lab. Entmol. Agrar. Filippo Silvestri 36:205-210.

HÉrard, F., Keller, M. A., Lewis, W. J., and Tumlinson, J. H. 1988. Beneficial arthropod behavior mediated by airborne semiochemicals: III. Influence of age and experience on flight chamber responses of Microplitis demolitor Wilkinson. J. Chem. Ecol. 14:1583-1596.

Hoballah Fritzsche, M. E., TAmò, C., and TurlingS, T. C. J. 2002. Differential attractiveness of induced odors emitted by eight maize varieties for the parasitoid Cotesia marginiventris: is quality or quantity important? J. Chem. Ecol. 28:951-968.

LEWIS, W. J. and TumLinson, J. H. 1988. Host detection by chemically mediated associative learning in a parasitic wasp. Nature 331:257-259.

Li, Y., Dickens, J. C., and SteInER, W. W. M. 1992. Antennal olfactory responsiveness of Microplitis croceipes (Hymenoptera: Braconidae) to cotton plant volatiles. J. Chem. Ecol. 18:1761-1773.

MAES, G. M. 1989. Catalogo de los insectos controladores biologicos en Nicaragua. Volumen III. Insectos parasitoides. Rev. Nicar. Entomol. 10:1-138.

MattiacCi, L., Dicke, M., and Posthamus, M. A. 1994. Induction of parasitoid attracting synomone in brussels sprouts plants by feeding of Pieris brassicae larvae: role of mechanical damage and herbivore elicitor. J. Chem. Ecol. 20:2229-2247.

MCAuslane, H. J., Vinson, S. B., and Williams, H. J. 1991. Influence of adult experience on host microhabitat location by the generalist parasitoid, Campoletis sonorensis (Hymenoptera: Ichneumonidae). J. Insect Behav. 4:101-113.

MCCALL, P. J., TURLINGS, T. C. J., LEWIS, W. J., and TuMLINSON, J. H. 1993. Role of plant volatiles in host location by the specialist parasitoid Microplitis croceipes Cresson (Braconidae: Hymenoptera). J. Insect Behav. 6:625-639.

Ngi-Song, A. J., Overholt, W. A., NJagi, P. G. N., Dicke, M., Ayertey, J. N., and Lwande, W. 1996. Volatile infochemicals used in host and host habitat location by Cotesia flavipes Cameron and Cotesia sesamiae (Cameron) (Hymenoptera: Braconidae), larval parasitoids of stemborers on graminae. J. Chem. Ecol. 22:307-323.

Powell, W., Pennacchio, F., Poppy, G. M., and Tremblay, E. 1998. Strategies involved in the location of hosts by the parasitoid Aphidius ervi Haliday (Hymenoptera: Braconidae: Aphidiinae). Biol. Control 11:104-112.

Reddy, G. V. P., Holopainen, J. K., and Guerrero, A. 2002. Olfactory responses of Plutella xylostella natural enemies to host pheromone, larval frass, and green leaf cabbage volatiles. J. Chem. Ecol. 28:131-143.

RÖSE, U. S. R., LEWIS, W. J., and TumLINSON, J. H. 1998. Specificity of systematically released cotton volatiles as attractants for specialist and generalist parasitic wasp. J. Chem. Ecol. 24:303-319.

SteinBerG, S., Dicke, M., and Vet, L. E. M. 1993. Relative importance of infochemicals from first and second trophic level in long-range host location by the larval parasitoid Cotesia glomerata. J. Chem. Ecol. 19:47-59.

Takabayashi, J., TAKahashi, S., Dicke, M., and Posthumus, M. A. 1995. Developmental stage of herbivore Pseudaletia separata affects production of herbivore-induced synomone by corn plants. J. Chem. Ecol. 21:273-287.

Turlings, T. C. J., Tumbinson, J. H., Lewis, W. J., and Vet, L. E. M. 1989. Beneficial arthropod behavior mediated by airborne semiochemicals: VIII. Learning of host-related odours induced 
by a brief contact experience with host by-products in Cotesia marginiventris (Cresson), a generalist larval parasitoid. J. Insect Behav. 2:217-226.

TurLings, T. C. J., TUMLInson, J. H., and LEWIS, W. J. 1990. Exploitation of herbivore-induced plant odours by host-seeking parasitic wasps. Science 250:1251-1253.

Turlings, T. C. J., WÄCKERS, F. L., Vet, L. E. M., Lewis, W. J., and TumLinson, J. H. 1993. Learning of host-finding cues by hymenopterous parasitoids, pp. 51-78, in D. R. Papaj et al. (eds.). Insect Learning Ecological and Evolutionary Perspectives. Chapman \& Hall, New York.

Turlings, T. C. J., Lengwiler, U. B., Bernasconi, M. L., and Wechsler, D. 1998. Timing of induced volatile emissions in maize seedlings. Planta 207:146-152.

Turlings, T. C. J., DAVISON, A., and TAMÒ, C. 2004. A six-arm olfactometer permitting simultaneous observation of insect attraction and odour trapping. Physiol. Entomol. 29:45-55.

VAN POECKE, R. M. P. and Dicke, M. 2002. Induced parasitoid attraction by Arabidopsis thaliana: involvement of the octadecanoid and the salicylic acid pathway. J. Exp. Bot. 53:1793-1799.

van Poecke, R. M. P., Posthumus, M. A., and Dicke, M. 2001. Herbivore-induced volatile production by Arabidopsis thaliana leads to attraction of the parasitoid Cotesia rubecula: chemical, behavioral, and gene-expression analysis. J. Chem. Ecol. 27:911-928.

Vet, L. E. M. and DiCKE, M. 1992. Ecology of infochemical use by natural enemies in a tritrophic context. Annu. Rev. Entomol. 37:141-172.

Vet, L. E. M. and Groenewold, A. W. 1990. Semiochemicals and learning in parasitoids. J. Chem. Ecol. 16:3119-3135.

VET, L. E. M., LEWIS, W. J., and CARDÉ, R. T. 1995. Parasitoid foraging and learning, pp. 65-101, in R. T. Cardé and W. J. Bell (eds.). Chemical Ecology of Insects 2. Chapman \& Hall, New York.

Whitman, D. W. and ElLer, F. J. 1990. Parasitic wasps orient to green leaf volatiles. Chemoecology 1:69-76.

Wickremasinghe, M. G. V. and VAn EMden, H. F. 1992. Reactions of adult female parasitoids, particularly Aphidius rhopalosiphi, to volatile chemical cues from the host plants of their aphid prey. Physiol. Entomol. 17:297-304.

ZANEN, P. O. and CARDÉ, R. T. 1991. Learning and the role of host-specific volatiles during in-flight host-finding in the specialist parasitoid Microplitis croceipes. Physiol. Entomol. 16:381-389. 